\title{
Drying and Hydrophobic Collapse of Paraffin Plates
}

\author{
Xuhui Huang, $\$$ Ruhong Zhou, \\ Department of Chemistry, Columbia University, New York, New York 10027 and Computational Biology \\ Center, IBM Thomas J. Watson Research Center, 1101 Kitchawan Road, Yorktown Heights, New York 10598
}

Received: October 1, 2004; In Final Form: December 2, 2004

\begin{abstract}
We perform molecular dynamics simulations of the hydrophobic collapse of two paraffin plates to examine how the collapse is mediated by realistic paraffin-water attractive van der Waals forces. We explore several aspects of the drying transition between the plates, including the critical separation for drying and the critical size of the vapor bubble required for the nucleation of the drying event. We also investigate the kinetics of hydrophobic collapse and find that the hydrophobic collapse occurs in about 100 ps. We compare these results with the simulations with the plate-water van der Waals attractions turned off and with recent results on the hydrophobic collapse of multidomain proteins. Last, we discuss the relationship among the dewetting transition critical distance, van der Waals potential well depth, and water contact angle on solute surface using a simple macroscopic theory.
\end{abstract}

\section{Introduction}

Understanding the hydrophobic interaction has long been recognized as the key to understanding many important problems, ${ }^{1}$ such as protein folding, ${ }^{2-5}$ self-assembly of amphiphiles, ${ }^{6}$ and capillary evaporation. ${ }^{7-10}$ Many biomolecules are characterized by surfaces containing extended nonpolar regions, and the aggregation and subsequent removal of waters between these hydrophobic surfaces is believed to be crucial. It is now widely known that small hydrophobic solutes hydrate differently than large ones. Small solutes such as methane can fit into the water hydrogen-bond network without destroying much of the hydrogen bonds, ${ }^{11,12}$ whereas larger hydrophobic solutes induce reorganization of water molecules. ${ }^{10,13,14}$ More interestingly, there might exist a drying layer as large as several water molecules around strongly hydrophobic surfaces, as first suggested by Stillinger ${ }^{15}$ and then studied by many other groups. ${ }^{6,9,16-28}$ In theoretical studies of these important phenomena it is often useful to invoke simple macroscopic theories or simplified computational models..$^{6,9,16-19,21,24,25}$ One particular such model is that the solute-water interaction is purely repulsive. ${ }^{29}$ Water dramatically reorganizes between such particles as they are brought together, so much so that for interparticle distances smaller than a certain critical distance, $D_{\text {c }}$, there can be a spontaneous drying transition that is a cavityinduced liquid-gas-phase transition ${ }^{30,31}$ where the interparticle region becomes devoid of water. ${ }^{29}$ When the solute-water interaction also has weak van der Waals attractions, the same phenomena are observed, although then $D_{\mathrm{c}}$ is expected to be smaller $^{25,29}$ the water depletion around single particles is either diminished or disappears completely. ${ }^{29}$ It is of interest to investigate how sensitive drying and depletion are to the strength of the solute-water attractions, especially for realistic solutewater attractions..$^{32,33}$

That attractive interactions can have a profound effect on these phenomena is clearly illustrated by recent molecular

* To whom correspondence should be addressed.

$\doteqdot$ Columbia University.

$\S$ IBM Thomas J. Watson Research Center. dynamics simulations of the collapse of a two-domain protein, the BphC enzyme, into a globular structure which examined how water molecules mediate hydrophobic collapse of proteins. ${ }^{34}$ Liquid water persisted in the interdomain region with a density $10-15 \%$ lower than in the bulk, even at small domain separations. Water depletion and hydrophobic collapse occurred on a nanosecond time scale, fully 1 or 2 orders of magnitude slower than that found by simulation in the collapse of idealized paraffin-like plates. When the electrostatic protein-water forces were turned off, a drying transition occurred in the interdomain region and the collapse speeded up by more than an order of magnitude. When attractive van der Waals forces were turned off as well, the dewetting in the interdomain region became more profound and the collapse became even faster. These findings show that realistic solute-water forces play a significant role in hydrophobic collapse. Clearly electrostatic forces between protein and water might be expected to be important. It is interesting that the van der Waals attractions play an important role as well.

To better understand the role of attractive van der Waals forces in hydrophobic collapse we extend our previous work on simple plates to two paraffin plates interacting with realistic van der Waals forces rather than the very weak forces in our previous publications. To make contact with the previous work ${ }^{29}$ we fit the Gay-Berne potential to explicit plates consisting of five parallel $n-\mathrm{C}_{18} \mathrm{H}_{38}$ hydrocarbon chains in the all-trans conformation, oriented parallel to the water interface. In this connection we mention the simulations of Jensen et $\mathrm{al}^{26}$ of depletion around paraffin with the molecules oriented perpendicular to the interface. In the present study of realistic paraffin plates we address the following questions.

(a) Given the stronger water-plate attractions, is there a drying transition for these realistic paraffin plates?

(b) If there is a drying transition, what is the critical size of the vapor cavity in the interplate region required for nucleation of dewetting when the separation between the plates is approximately the critical separation?

(c) For the realistic case of paraffin plates will we observe the same kinetic mechanism for collapse that we observed for 
a.
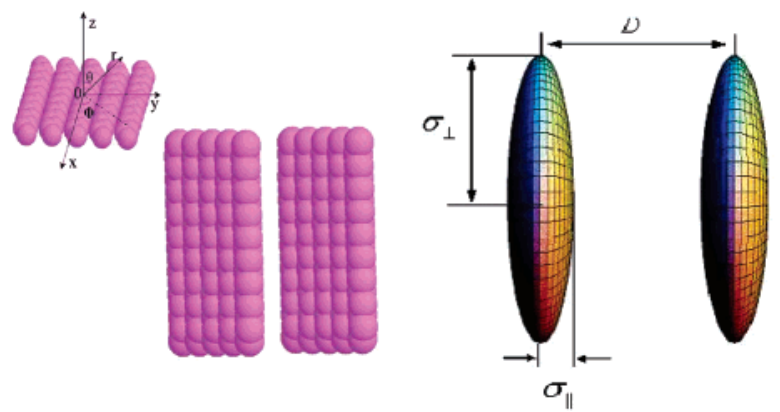

c.

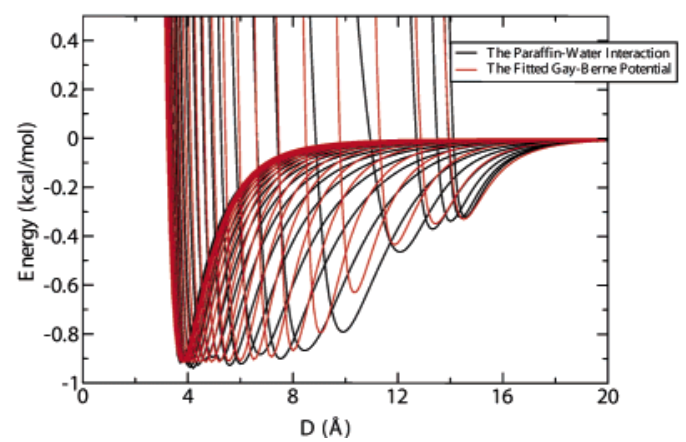

Figure 1. (a) Schematic diagram for the two paraffin plate system. Each plate consists of five $n-\mathrm{C}_{18} \mathrm{H}_{38}$ molecules. The coordinate system is shown in the insert. (b) Schematic diagram for the two ellipsoids system in which parameters $D, \sigma_{\perp}$, and $\sigma_{\|}$are defined. (c) The OPLSUA/ SPC paraffin-water potential (black) and the fitted Gay-Berne potential (red) are compared in this graph. The OPLSUA/SPC paraffinwater potential curves correspond to moving a water molecule along the $\vec{r}$ axis in the $x z$ plane with an angle $\theta$ away from one paraffin plate. The Gay-Berne potential curves, on the other hand, are plotted from eq 1 for each angle $\theta$. The angles $\theta$ are plotted every $4^{\circ}$ from $0^{\circ}$ to $90^{\circ}$.

the idealized case where the plate-water interaction was purely repulsive. That is, do we see the region between the approaching plates suddenly dry after which the plates quickly collapse?

(d) How do the kinetics of hydrophobic collapse depend on whether plate-plate attractions are present or absent? Will the collapse be significantly faster with plate-plate attractions on?

(e) When can we expect to see dewetting? For example, if for a paraffin plate we see it, should we also see it for an equalsized graphite plate. Interestingly, macroscopic theory predicts that we should not.

\section{System and Methods}

The paraffin plate in this study is fit from five $n-\mathrm{C}_{18} \mathrm{H}_{38}$ molecules which are aligned in parallel in one plane (the crystal structure of $n-\mathrm{C}_{18} \mathrm{H}_{38}$ taken from Cambridge Crystallographic Database $^{35}$ ), as shown in Figure 1a. Two such identical paraffinlike plates are then solvated in SPC water. ${ }^{36}$ The Gay-Berne potential is used for the interaction between the ellipsoidal plates and water (oxygen atom of water) and between the two plates ${ }^{29,37-39}$ with parameters determined by a similar procedure described elsewhere. ${ }^{29,38,39}$

The OPLSUA force field is first adopted for the $n-\mathrm{C}_{18} \mathrm{H}_{38}$ molecules. ${ }^{40}$ The $\mathrm{CH}_{3}$ and $\mathrm{CH}_{2}$ groups are modeled as uncharged Lennard-Jones $(\mathrm{LJ})$ particles. For the $\mathrm{CH}_{3}$ group $\sigma_{\mathrm{CH} 3 \mathrm{CH} 3}=$ $3.905 \AA$ and $\epsilon_{\mathrm{CH} 3 \mathrm{CH} 3}=0.175 \mathrm{kcal} / \mathrm{mol}$. For the $\mathrm{CH}_{2}$ group $\sigma_{\mathrm{CH} 2 \mathrm{CH} 2}=3.905 \AA$ and $\epsilon_{\mathrm{CH} 2 \mathrm{CH} 2}=0.118 \mathrm{kcal} / \mathrm{mol}{ }^{40}$ The mass of each plate is chosen to be the mass of five $n-\mathrm{C}_{18} \mathrm{H}_{38}$ molecules. The Gay-Berne water-plate interaction is then fit to the water-paraffin potential with angle $\phi=0^{\circ}$ (see Figure 1a). The water-plate Gay-Berne interaction is taken to be

$$
\begin{aligned}
& U_{\mathrm{pw}}= \\
& 4 \epsilon\left[\left(\sigma_{0} /\left(r-\sigma(\chi ; \theta)+\sigma_{0}\right)\right)^{12}-\left(\sigma_{0} /\left(r-\sigma(\chi ; \theta)+\sigma_{0}\right)\right)^{6}\right]
\end{aligned}
$$

where $\sigma(\chi ; \theta)=\sigma_{\perp}\left(1-\chi \cos ^{2} \theta\right)^{-1 / 2}$ and $\chi=\left(\sigma_{\|}^{2}-\sigma_{\perp}^{2}\right) / \sigma_{\|}^{2}$. $\chi, \sigma_{\|}$, and $\sigma_{\perp}$ are the axial ratio and lengths of the oblate ellipsoid along the major and minor axes, respectively (see Figure 1b); $\theta$ is the angle between the major axis of the ellipsoid and the unit vector pointing from the center of the ellipsoid to water's oxygen atom, as shown in Figure 1a. In the water-plate GayBerne potential, the dependence of $\epsilon$ on $\chi$ and angle $\theta$ has been fitted to a function $\epsilon=\left(0.9161-0.5957 /\left(1+e^{-0.9982(\sigma(\gamma ; \theta)-9.8703)}\right)\right)$, with parameters $\sigma_{\perp}, \sigma_{\|}$, and $\sigma_{0}$ equal to $14.1,3.2$, and $4.6 \AA$, respectively. Both the best-fitted water-plate Gay-Berne potential and the original OPLSUA/SPC water-paraffin potential versus different angles $\theta$ are displayed in Figure 1c. The fitted potential curves reproduce the original force field potentials reasonably well.

The Gay-Berne potential (see eq 1) is also used to fit the interaction between the two paraffin plates. $\epsilon$ and $\sigma$ in eq 1 are redefined ${ }^{37}$ to be

$$
\epsilon=\epsilon_{0}\left[1-\chi^{2}\left(\mathbf{u}_{1} \cdot \mathbf{u}_{2}\right)^{2}\right]^{-1 / 2}
$$

and

$$
\sigma=\sigma_{0}\left(1-\frac{1}{2} \chi\left[\frac{\left[\hat{\mathbf{r}} \cdot\left(\mathbf{u}_{1}+\mathbf{u}_{2}\right)\right]^{2}}{1+\chi\left(\mathbf{u}_{1} \cdot \mathbf{u}_{2}\right)}+\frac{\left[\hat{\mathbf{r}} \cdot\left(\mathbf{u}_{1}-\mathbf{u}_{2}\right)\right]^{2}}{1-\chi\left(\mathbf{u}_{1} \cdot \mathbf{u}_{2}\right)}\right]\right)^{-1 / 2}
$$

where $\mathbf{u}_{1}$ and $\mathbf{u}_{2}$ are, respectively, the unit vectors along the major axis of ellipsoid 1 and ellipsoid 2. $\hat{\mathbf{r}}$ is the unit vector pointed from the center of ellipsoid 1 to the center of ellipsoid 2. $\chi$ has the same definition as in eq 1 . The fitted values of $\sigma_{\perp}$, $\sigma_{\|}, \sigma_{0}$, and $\epsilon_{0}$ are $5.3 \AA, 4.2 \AA, 4.6 \AA$, and $73.9 \mathrm{kcal} / \mathrm{mol}$, respectively, when the two paraffin-like plates are parallel.

In the following simulations a position restraint potential is also applied to force the plates to remain parallel to the $X-Y$ plane

$$
V_{\mathrm{pr}}=(1 / 2) k_{\mathrm{pr}}\left(x_{i}^{2}+y_{i}^{2}\right)
$$

where $i$ labels each of the two points on the major axis of each ellipsoid. The major axis of the ellipsoids was chosen to be along the laboratory $Z$ axis, and $k_{\mathrm{pr}}$ is the force constant, 5000 $\mathrm{kcal} / \mathrm{mol} \AA^{2}$. In addition to this plane restraint force there is another restraint applied to prevent the plates from diffusing too far apart in kinetics studies

$$
V_{\mathrm{dr}}=\left\{\begin{array}{ll}
k_{\mathrm{dr}}\left(D-D_{0}\right)^{6} & \text { for } D>D_{0} \\
0 & \text { for } D \leq D_{0}
\end{array}\right\}
$$

where $D$ is the distance between centers of the plates, $k_{\mathrm{dr}}=$ $1 \mathrm{kcal} / \AA^{6}$, and $D_{0}$ was chosen to be $20 \AA$ in this constraint.

The simulations were performed at constant temperature $(298.15 \mathrm{~K})$ and pressure $(1 \mathrm{~atm})$ with Nose-Hoover chain (NHC) thermostats and an Andersen-Hoover-type barostat ${ }^{41,42}$ using the program SIM. ${ }^{43}$ The RATTLE ${ }^{44}$ algorithm was used to constrain the internal geometry of the water molecules, and the particle-particle particle-mesh Ewald method ${ }^{45,46}$ was used to treat long-range electrostatic interactions. A box with 2048 water molecules was first equilibrated for $150 \mathrm{ps}$, then the ellipsoids were introduced, and the overlapping water molecules 
were removed. A series of simulations were performed to study both the thermodynamic and kinetic behaviors of the paraffinplate water system.

One caveat that should be noted is that NPT MD introduces fictitious dynamics both from the temperature and pressure control. New fictitious degrees of freedom are introduced with corresponding kinetic energies, fictitious masses, and fictitious frequencies. These influence the time scales and can affect the rate processes observed. We are presently studying these issues. Preliminary work comparing NPT and NVE ensembles suggests that kinetic time scales in the NPT dynamics can be different than in a suitably equivalent NVE ensemble, but the kinetic mechanisms might be very similar. For drying dynamics we are comparing the NPT ensemble to an NVE ensemble in which the plates are dispersed in a sheet of water surrounded by vapor on either side. Thermodynamic and structural equilibrium properties are expected to be the same for the two ensembles.

\section{Results and Discussion}

It is known from previous simulations as well as macroscopic theory that $D_{\mathrm{c}}$, the critical separation for drying between the plates, will decrease as the strength of the van der Waals attractive interaction is increased. ${ }^{29}$ For example, for parallel cylindrical plates it has been shown ${ }^{29}$ that when the contact angle, $\theta_{\mathrm{c}}$, for water in contact with the plate is obtuse, $D_{\mathrm{c}} \propto$ $\cos \theta_{\mathrm{c}}$ (with similar results for parallel oblate ellipsoidal plates (Gay-Berne plates)). Since the contact angle decreases as the strength of the attraction between water and the plate increases, $D_{\text {c }}$ should also decrease. For sufficiently strong attractions we thus expect $\theta_{\mathrm{c}}$ to eventually become acute, at which point no drying transition will occur. In fact, for obtuse contact angles close to $90^{\circ}$ drying may be possible in principle but not in practice because the predicted $D_{\mathrm{c}}$ gets so small that steric effects will not allow a layer of water to fit between the plates. Drying is thus very sensitive to the strength of the attractive forces. For example, at room temperature the contact angle of water on graphite is known to be acute so that, if macroscopic theory is valid down to such small separations, drying should not occur. What is the situation for paraffin, the quintessential waxy hydrophobic substance? In a previous paper $^{29}$ we studied fictitious Gay-Berne plates in water, but we chose arbitrary parameters for the force field which in retrospect correspond to a much weaker water-plate attraction than for paraffin. Our previous work correlated very well with the predictions of macroscopic theory both for $D_{\mathrm{c}}$ and for the free energy barriers to nucleation of a vapor bubble in the drying phenomenon. Here we focus on simulating something akin to real paraffin, although the Gay-Berne model, fit as we describe above, may still be a significant oversimplification. In addition, the foregoing discussion is predicated on the validity of macroscopic theory, a theory that may well breakdown at some point for the small systems described in this paper, despite the fact that our foregoing results on idealized plates seem to correlate with macroscopic theory.

Two 200 ps simulations with different initial conditions were launched for each given interplate distance $D$ from 6.5 to 15 $\AA$, one simulation with water initially filling the interplate region ("wet" initial condition) and the other with water initially removed from this region ("dry" initial condition). Results for interplate distances $D=7,9$, and $11 \AA$ are displayed in Figure 2a, b, and c, respectively. In the simulation of $D=7 \AA$ with wet initial conditions (see the first row of Figure 2a), large fluctuations were observed in the region between the plates. At about 100 ps a large cavity forms in this region. The system then dries, and it remains dry for the rest of the simulation. a.
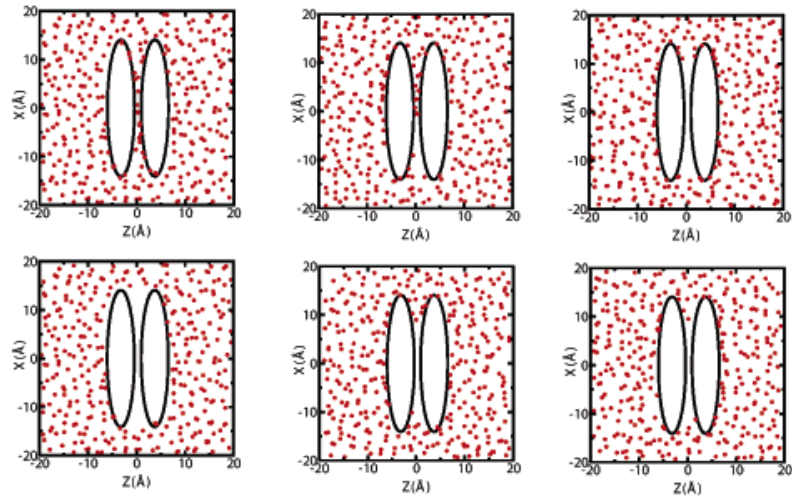

b.
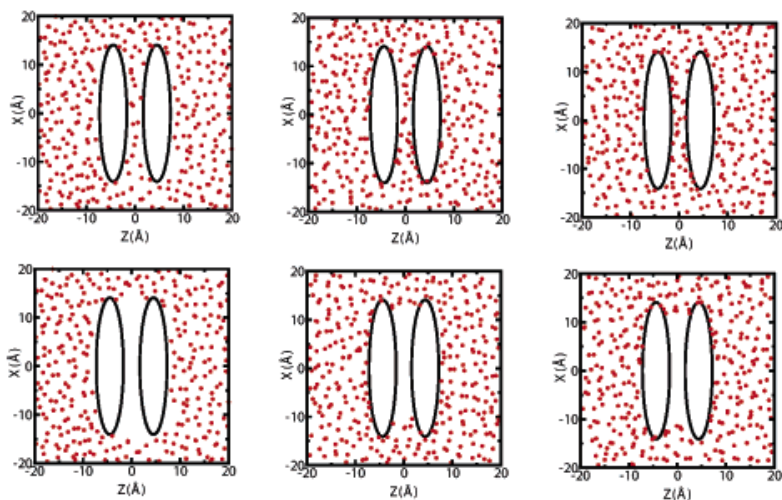

C.
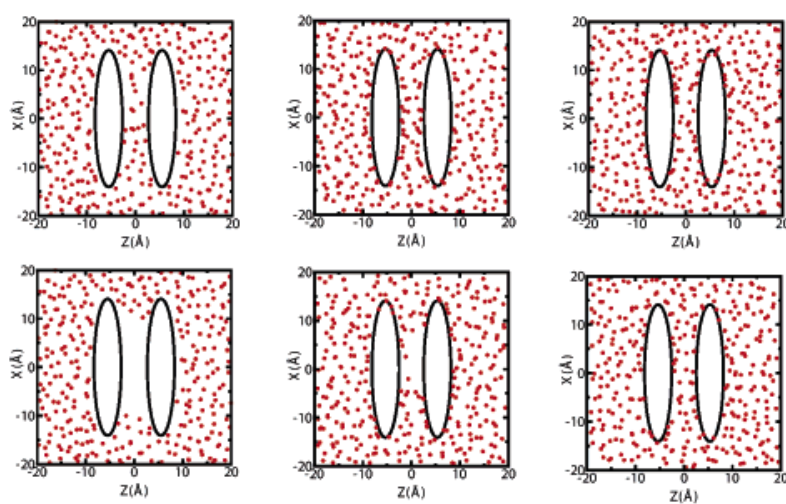

$\mathrm{t}=0 \mathrm{ps}$
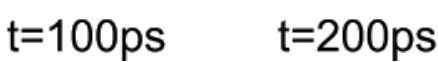

Figure 2. (a) Snapshots corresponding to the time evolution of the system with an interplate distance of $D=7 \AA$. The two-dimensional projections of the two-plate system in SPC water are used. Red dots correspond to the positions of water oxygen atoms. Water molecules obscuring the solute (i.e., those water molecules in which the absolute value of the $Y$ coordinate, which in this case is perpendicular to the projection plane, is greater than $4 \AA$ ) have been removed. The system starts from both dry and wet initial configurations. (b) Same projections as part a with an interplate distance $D=9 \AA$. (c) Same as part a with an interplate distance $D=11 \AA$.

When dry initial conditions are deployed instead, the system remains dry for the entire 200-ps simulation (see the second row of Figure 2a). Thus, the dry state is found to be the thermodynamically more stable state for $D \leq 7 \AA$. In the current system $D=6.4 \AA$ is the smallest interplate distance for which one layer of water molecules can fit sterically between the two plates. When the interplate distance $D \geq 11 \AA$, the "wet" state becomes the thermodynamically more stable state, as shown in Figure 2c. In this case, when the system starts from the "dry" 
a.
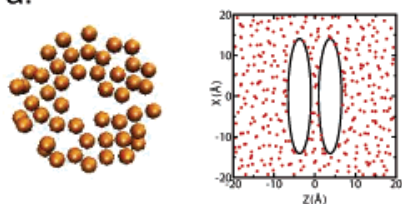

$\mathrm{t}=0 \mathrm{ps}$

b.

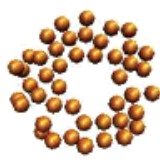

$r=4 \AA$

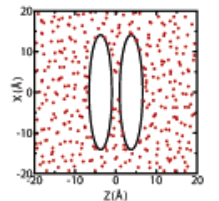

$\mathrm{t}=0 \mathrm{ps}$

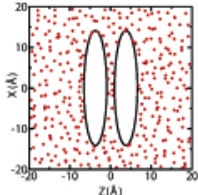

$\mathrm{t}=10 \mathrm{ps}$
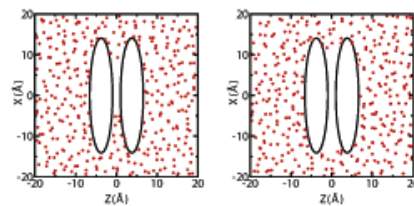

$\mathrm{t}=32 \mathrm{ps}$

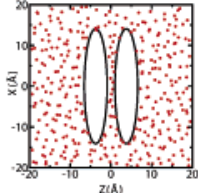

$t=100$ ps

Figure 3. (a) (far left) Initial water configuration in the region between the two plates. Water molecules in the cylindrical region with radius $r=11$ A between the two plates are projected on the $X Y$ plane. Each ball corresponds to the position of a water oxygen atom. This initial configuration was prepared with a cylindrically shaped vapor cavity with radius $r=3 \AA$. The other three figures display the time evolution of the simulation system. Projections were constructed in the same way as in Figure 2. (b) Same as part a with cavity radius $r=4 \AA$.

initial condition, water quickly refills the interplate gap in about $100 \mathrm{ps}$. When the system starts from the "wet" initial condition, it remains wet for the entire 200-ps simulation. For cases with $D$ ranging from 7 to $11 \AA$, hysteresis is observed in the 200-ps run, that is, simulations starting from "wet" initial conditions remain wet and simulations starting from "dry" initial conditions remain dry (see Figure 2b). Large fluctuations are also observed in all of these simulations. We conclude that the critical distance for paraffin plates, of the size studied and under the thermodynamic conditions simulated, lies somewhere in the range $7 \AA \leq D_{\mathrm{c}} \leq 11 \AA$.

The simple macroscopic theory ${ }^{29}$ predicts, and simulations confirm, that there are free energy barriers for the transitions wet $\rightarrow$ dry and dry $\rightarrow$ wet. These barriers can become quite large at $D \approx D_{\mathrm{c}}$. Thus, it is not unexpected to observe hysteresis in the paraffin plates. When $7 \AA \leq D \leq 11 \AA$, fluctuations are observed for plates started from the "wet" initial condition and small vapor cavities arise and regress in the interplate region but the system still remains partially wet. In order for the system to pass over the free energy barrier to become "dry", vapor cavities of a critical size must grow to nucleate the dewetting of the interplate region. A series of 100-ps simulations were performed to study the critical size of the vapor cavity at different $D$. Initial configurations were prepared with different sized cylindrically shaped vapor cavities between the two plates (radii $r=3,4, \ldots, 11 \AA$ ) and with an interplate separation fixed at $D=7.5 \AA$. In the simulation with $r=3 \AA$, as shown in Figure $3 \mathrm{a}$, it takes about $10 \mathrm{ps}$ for the water to fill up the cavity and the system stays wet for the rest of the simulation. When the initial cavity size $r$ is increased to $4 \AA$, the cavity grows and the interplate region dries in about $100 \mathrm{ps}$, as shown in Figure $3 \mathrm{~b}$. When the cavity size $r$ is greater than $4 \AA$, the interplate region dries. These results indicate that for an interplate distance $D=7.5 \AA$, the critical vapor cavity radius for the nucleation of a vapor bubble and thus for the wet $\rightarrow$ dry transition is between 3 and $4 \AA$ for plates of radius $14.1 \AA$.

The critical cavity size $r_{\mathrm{c}}$ is also computed for other interplate distances. When $D=8.5$ and $10.0 \AA, r_{\mathrm{c}}$ is found to be between 4 and $5 \AA$ and between 6 and $7 \AA$, respectively. When $D \geq 11$ $\AA, r_{\mathrm{c}}$ does not exist because even if a vapor cavity as large as the entire interplate region is initiated, the system still returns to the "wet" state. The existence of a critical vapor cavity radius

a.

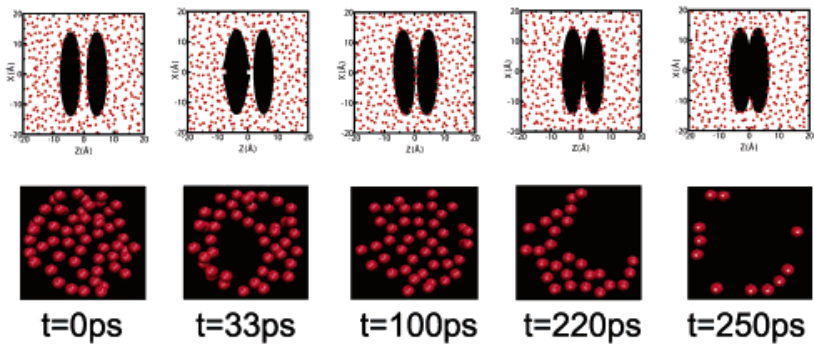

b.

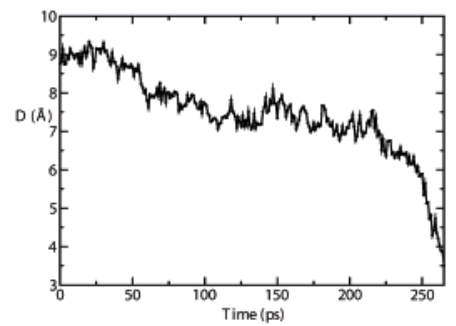

Figure 4. (a) Snapshots corresponding to the time evolution of the system which has no direct interaction between the two plates. In the first row projections were constructed in the same way as in Figure 2. Plates were constrained so they could only move along the $z$ direction. The initial interplate distance is $D=9 \AA$. The second row shows the snapshots of the water configuration in the region between the two plates. (b) Interplate distance $D$ (the distance between the centers of the two ellipsoids in $\AA$ ) as a function of simulation time.

means that the "wet" and "dry" states are separated by a free energy barrier. The system can stay in either of the two states for a long time until activated. To determine which state is the thermodynamically more stable state for a given separation near $D \approx D_{\text {c }}$, a long MD simulation would be required to generate enough statistics to determine the probabilities of the "wet" and "dry" states.

Despite the attractive water-plate van der Waals interactions in paraffin, we observe a drying transition, as expected from macroscopic theory with the experimental contact angle for paraffin. The question now is will we observe the same twostep mechanism for hydrophobic collapse that we previously observed for the idealized hydrophobic plates with purely repulsive plate-water interactions? That is, does the interplate region suddenly dry before the plates collapse? To answer this question we generated eight molecular dynamics trajectories each starting at an initial interplate distance of $D=9 \AA$ and each of length $100 \mathrm{ps}$. The initial configurations for these eight trajectories were taken at intervals of $t=20 \mathrm{ps}$ after the first $40 \mathrm{ps}$ from the previous $200 \mathrm{ps}$ trajectory for plate separation fixed at $D=9 \AA$ (as shown in the first row of Figure $2 \mathrm{~b}$ ). In these simulations the direct plate-plate interaction is turned off. Plates were only allowed to move along the $Z$ axis and also precluded from drifting apart to distance larger than $20 \AA$ (approximately one-half of the box length). In six of the eight 100-ps trajectories the plates just diffuse around the initial separation, sometimes even diffusing apart. Since plates do not directly interact with each other in these simulations, the motion of the plates is only driven by their hydrophobic response to the solvent. Presumably the plates would collapse if the trajectories were continued beyond 100-ps simulations for some of these trajectories. In the remaining two trajectories the two paraffin plates do indeed collapse. Each of these later two trajectories were then extended for an additional $200 \mathrm{ps}$ to generate more data. Snapshots as time progresses are shown in Figure $4 \mathrm{a}$, where it can be seen that initially there is more than 
a.
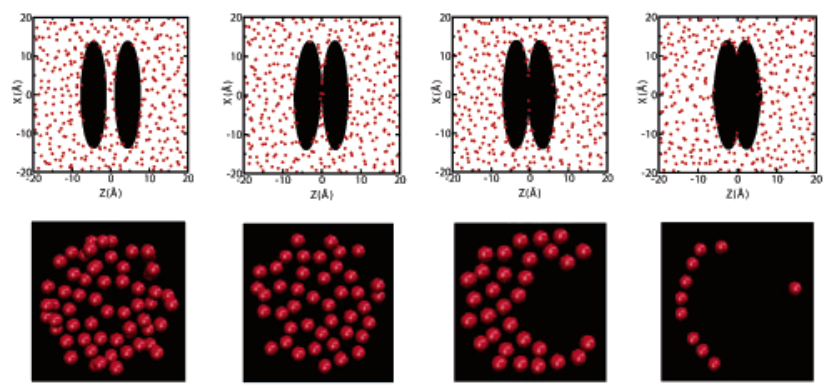

$\mathrm{t}=30 \mathrm{ps}$

$\mathrm{t}=69 \mathrm{ps}$

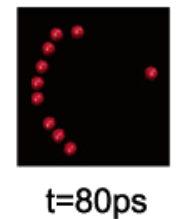

b.

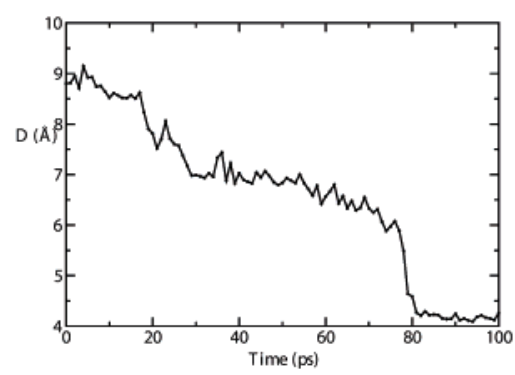

Figure 5. (A) Snapshots corresponding to the time evolution of the system which has direct interaction between the two plates. In the first row projections were constructed in the same way as in Figure 2. Plates were constrained so they could only move along the $z$ direction. Initial interplate distance was $D=9 \AA$. The second row shows the snapshots of the water configuration between the two plates. (B) Interplate distance $D$ (the distance between the centers of the two ellipsoids in $\AA$ ) as a function of time.

a monolayer of water between the two plates. As time progresses bubbles arise but then regress until a large enough bubble forms at approximately $t=220 \mathrm{ps}$, and then the full drying transition takes place between the two plates. Thereafter the two plates collapse quickly and come into contact. Figure $4 \mathrm{~b}$ shows the time evolution of the plate-plate separation corresponding to the snapshots in Figure 4a. During the period when the bubbles arise and regress the separation between the two plates diffuses around $D=7.2 \AA$, but because of the stronger paraffin-water attractions the behavior is somewhat slower compared to the idealized plates. In the case of the idealized plates the critical separation is larger and it is easier to observe the two-speedlike collapse than when attractions are present. Furthermore, the simple macroscopic theory might be more applicable for the idealized plates than for the realistic paraffin plates because more water molecules fill the interparticle gap in the former than in the later (where only a monolayer of water is involved).

In the foregoing simulations the plates interact with water but not with each other. How do direct interactions between the plates alter the time course of the collapse? To answer this we repeat the foregoing simulations by including the GayBerne plate-plate interactions where the parameters were determined as outlined in section 2. Starting from the same eight initial configurations as described above (i.e., without direct plate-plate interactions), we find that in all eight trajectories the two plates collapse in less than $100 \mathrm{ps}$. The result for a typical trajectory is shown in Figure 5. Comparing the snapshots here with those of Figure 4 we see that when the plates attract each other directly the collapse speeds up. Initially the plates are separated by more than one monolayer of water. In approximately $30 \mathrm{ps}$ they become separated by essentially only one monolayer of water. This process is much faster, by almost a factor of 3, than in the earlier case where the plates do not interact with each other. This is probably because the two plates

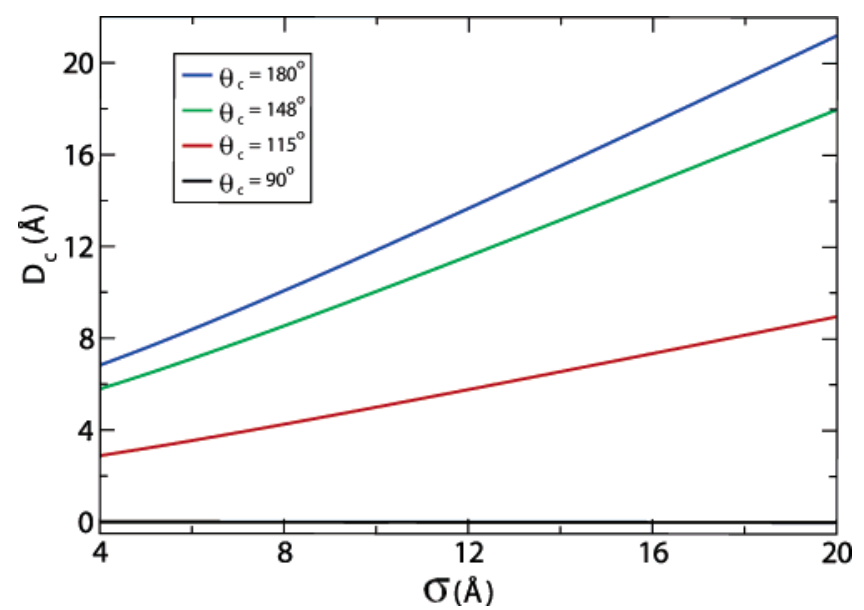

Figure 6. Critical distance versus the radius $\left(\sigma_{\perp}\right)$ of the ellipsoid plates with different water contact angle. The curves are predicted from macroscopic theory.

are executing forced diffusion under an attractive force (described by the Smolochwski equation ${ }^{47}$ ). Thereafter a twodimensional bubble forms and grows with no observable regression. The remainder of the collapse looks very similar to the case when there is no attractive force between the plates, i.e., a cavity grows and reaches critical size, the region dries, and the plates fully collapse. In all of the eight trajectories this whole process takes about $50-100 \mathrm{ps}$, again between two to three times faster than in the case of the noninteracting plates. The attractive interaction between the plates speeds up the diffusion of the plates to the critical separation and results in hydrophobic collapse for all eight trajectories, while in the corresponding simulations for plates without plate-plate interactions, only two out of eight were observed to collapse in the given simulation time. Clearly, if the attractive plate-plate forces are strong enough, vapor cavities might be forced to form rather than arising from spontaneous thermal fluctuations.

It is interesting to note that both the critical distance and the collapse time of the paraffin-like plates are similar to the twodomain protein $\mathrm{BphC}$ when the protein-water electrostatic interaction is turned off, ${ }^{34}$ even though the protein system is much more complex. In the two-domain protein case with the protein-water electrostatic interactions turned off (Turnoff1 option in ref 34) the critical distance is found to be between 7 and $9 \AA$ and the two-domain collapse time is approximately 150 ps.

From the foregoing simulations a simple picture emerges. Drying-induced collapse occurs between waxy plates such as paraffin. Will it be seen in graphite under similar conditions in which liquid water coexists with its vapor? It is interesting to note that in recent computational studies by Hummer and coworkers of water inside a carbon nanotube and by Pettitt and co-workers ${ }^{48,49}$ of water between two graphite-like plates dewetting was not observed. In these studies the solute-solvent attraction is approximately 2 times stronger than in our paraffinlike plates and approximately 4 times stronger than in our previous work on idealized hydrophobic plates ${ }^{29}$ (with the same fitting procedure as described in section 2 , the well depth in the Gay-Berne potential will be $-1.83,-0.91$, and $-0.42 \mathrm{kcal} /$ mol for graphite plates, paraffin plates, and previous idealized plates, respectively). As mentioned earlier, a simple macroscopic thermodynamic model based on Young's equation allows one to approximately predict the critical distance for drying. ${ }^{29}$ This critical distance depends on the size of the ellipsoidal plates and the contact angle between the plate and water. Figure 6 
gives plots of the critical distance $D_{\mathrm{c}}$ versus the radius of the plate for different contact angles predicted by macroscopic theory. For a given plate size $\sigma_{\perp}, D_{\mathrm{c}}$ decreases as the contact angle gets smaller. The larger the contact angle, the greater will be the critical distance. On the other hand, the larger the attractive interaction between the plates and water, the smaller will be the contact angle. From this plot it can be seen that for small, yet obtuse, contact angles, that is, for large values of the LJ parameter $\epsilon$, the critical distance can be too small to allow for any dewetting. Clearly, dewetting will disappear when the critical distance is too small to even allow one layer of water to fit into the interplate region at the critical separation. Interestingly, Lundgren ${ }^{50}$ et al. recently studied the water contact angle versus the Lennard-Jones potential well depth $\epsilon$ between the solute and solvent. They also calculated the contact angle for the graphite surface, $\theta_{\mathrm{c}}=83^{\circ}$, which agrees well with the experimental value of $\theta_{\mathrm{c}}=86^{\circ}$, an acute contact angle. For this case macroscopic theory predicts that drying will not occur for any size graphite plates. Two graphite monolayer plates must be approximately at least $6.2 \AA$ apart to accommodate one layer of water. Thus, no dewetting transition should be observable for graphite plates. On the other hand, the contact angle will be about $115^{\circ}$ for our paraffin-like plates from Lundgren's data (Figure 8 in ref 50), which gives a critical distance of about $\sim 7 \AA$, in excellent qualitative agreement with our above findings. The contact angle will further increase to about $148^{\circ}$ for the previous idealized full-potential hydrophobic plates, and the predicted critical distance of $\sim 13 \AA$ also agrees very well with our previous findings from computer simulation. ${ }^{29}$ For purely repulsive hydrophobic plates the contact angle will then approach $180^{\circ}$, and the critical distance will be even greater for the same plate size. Thus, in conclusion, the nonexistence of drying in carbon nanotubes or graphite-like plates is probably because they attract water much more strongly than the paraffin plates, with a resulting water-graphite contact angle near $90^{\circ}$ and a very small critical distance (if one exists) that cannot accommodate even one layer of water molecules. Needless to say, the macroscopic theory is likely to break down for the prediction of small critical distances because so few water molecules are involved.

Finally, we simulate all-atom paraffin molecule plates containing five $\mathrm{C}_{18} \mathrm{H}_{38}$ molecules as shown in Figure 1a. Here the molecules in the plates are taken to be parallel to the platewater interface instead of perpendicular to the interface as in a recent publication. ${ }^{26}$ At each interplate distance $D$ (defined as the closest distance between the nucleus of $\mathrm{C}$ atoms in the two paraffin molecule plates) two 500-ps simulations are performed with "wet" and "dry" initial conditions, respectively. The critical distance range for this molecule plate system is found to be between 6.9 and $7.5 \AA$. When $D \leq 6.9 \AA$, a complete dewetting is observed. $D=6.4 \AA$ is the smallest distance for water to fit between the two molecule plates sterically. This observation is consistent with the results of the fitted Gay-Berne plates described above. The critical distance range for this system, $6.9 \AA \leq D \leq 7.5 \AA$, is somewhat smaller than that in the fitted Gay-Berne plates system, $7 \AA \leq D \leq 11 \AA$. This difference in $D_{\text {c }}$ range may be due to the rough surface of the all-atom paraffin plates made by five $\mathrm{C}_{18} \mathrm{H}_{38}$ molecules. The troughs between paraffin molecules in each plate form more attractive regions for water molecules to occupy, which might effectively reduce the upper bound of the critical distance. These troughs can act as "traps" for water molecules for $D>7.5 \AA$, as observed in the trajectory movies.

To investigate the sensitivity of dewetting to the strength of
TABLE 1: Existence of the Dewetting Phenomenon for the Graphite-like Systems with Various Solute-Solvent Interactions

\begin{tabular}{ccccc}
\hline & \multicolumn{3}{c}{$\begin{array}{c}\text { equivalent well depth of } \\
\text { water-plate interaction } \\
(\mathrm{kcal} / \mathrm{mol})\end{array}$} & $\begin{array}{c}\epsilon_{\mathrm{CO}} \\
\text { case no. }\end{array}$ \\
\hline 1 & 0.0860 & 0.1156 & -1.83 & nowetting \\
2 & 0.0521 & 0.0900 & -1.43 & no \\
3 & 0.0361 & 0.0749 & -1.19 & no \\
4 & 0.0299 & 0.0682 & -1.09 & no \\
5 & 0.0269 & 0.0647 & -1.02 & $a$ \\
6 & 0.0240 & 0.0611 & -0.97 & yes \\
7 & 0.0213 & 0.0575 & -0.91 & yes \\
8 & 0.0120 & 0.0432 & -0.68 & yes \\
9 & 0.0054 & 0.0290 & -0.45 & yes
\end{tabular}

${ }^{a}$ In this case water dewets and then refills the interplate region during 500 ps simulations.

solute-solvent attractions we also perform a series of simulations with previously studied all-atom graphite systems ${ }^{48,49}$ in SPC water using different water-carbon interactions. Each graphite plate has 170 carbon atoms with $\mathrm{C}-\mathrm{C}$ bond lengths of $1.4 \AA$, and the size of the plate $(r \approx 14 \AA$ ) is similar to the paraffin plate described in Figure 1a. The interplate distance is fixed to be $6.8 \AA$, but the strength of the water-plate interaction varies. A series of 500-ps simulations starting from "wet" initial conditions is performed for different water-carbon interactions. The results are displayed in Table 1 . No drying was observed with the normal graphite potential ${ }^{48}$ (case 1 in Table $1, \epsilon_{\mathrm{CC}}=$ $0.086 \mathrm{kcal} / \mathrm{mol}$ and $\left.\epsilon_{\mathrm{CO}}=0.1156 \mathrm{kcal} / \mathrm{mol}\right)$, which is consistent with the above contact angle analysis and previous simulations. ${ }^{49}$ The equivalent Gay-Berne potential well depth of the watergraphite plate interaction is estimated to be about $-1.83 \mathrm{kcal} /$ mol. The reason we use the Gay-Berne well depth for comparison is because we cannot simply compare the van der Waals $\epsilon_{\mathrm{CC}}$ alone due to the different compactness of $\mathrm{C}$ atoms in graphite or paraffin molecules $-\mathrm{C}$ atoms in graphite are more compact than those in the paraffins. However, when the watercarbon interaction $\epsilon_{\mathrm{CO}}$ is decreased to $0.0611 \mathrm{kcal} / \mathrm{mol}\left(\epsilon_{\mathrm{CC}}=\right.$ $0.024 \mathrm{kcal} / \mathrm{mol}$ ), dewetting is observed in a 500 -ps simulation (case 6 in Table 1). When the water-carbon interaction is even weaker, the system will always dry. Interestingly, the GayBerne potential well depth of the paraffin plate is about -0.91 $\mathrm{kcal} / \mathrm{mol}$, which is equivalent to case 7 in Table 1 . Dewetting is expected and also observed in this case, a result consistent with the conclusion drawn from the paraffin plate simulations. It is interesting to note that when $\epsilon_{\mathrm{CO}}=0.0647 \mathrm{kcal} / \mathrm{mol}$ the system first dries and then wets (water refills the interplate region) during the 500-ps simulations. A much longer simulation also shows the system fluctuates between the "dry" and "wet" states, indicating that $D=6.8 \AA$ is roughly the critical distance for the graphite system with $\epsilon_{\mathrm{CC}}=0.027 \mathrm{kcal} / \mathrm{mol}$ (case 5 in Table 1).

\section{Conclusion}

Molecular dynamics simulations of two Gay-Berne paraffin plates in water are consistent with there being a critical distance $D_{\text {c }}$ such that when the plates are brought together closer than $D_{\text {c }}$ spontaneous drying takes place. This conclusion is based on the following observations. For plate separations $D \leq 7 \AA$ (with plates fixed in space), the system starting from both "dry" and "wet" initial conditions ends up in the "dry" state. On the other hand, when $D \geq 11 \AA$, the final state is always observed to be the "wet" state, regardless of the initial condition. For separations close to the critical (7 $\AA \leq D \leq 11 \AA$ ), hysteresis is observed, i.e., starting from "wet" initial condition the system 
remains wet, and starting from "dry" initial condition the system remains dry. This is consistent with macroscopic theory, ${ }^{29}$ which predicts that the free energy barrier between the "wet" and "dry" states will be large when plate separation is close to the critical distance $D_{\text {c }}$. Simulations show that when the system is prepared with a large enough cavity inside the interplate region it can more easily cross the free energy barrier and dry. For example, the critical cavity sizes corresponding to interplate distances $D=7.5,8.5$, and $10 \AA$ are found from simulation to be between 3 and $4 \AA$, between 4 and $5 \AA$, and between 6 and $7 \AA$, respectively. Simulations on all-atom paraffin plates display the same features as seen in the Gay-Berne plates.

In the kinetics of collapse of the two paraffin-like plates the plates first diffuse together to near the lower estimate of the critical distance $D_{\mathrm{c}} \approx 7 \AA$. Then a drying transition takes place, expelling the last layer of water, at which point the plates completely collapse. This happens with and without attractive interactions between the plates. When the plates attract each other the initial diffusion process is biased by the attractive force, so that the plates get close faster, but after that the kinetics of collapse look very similar for the attracting plates as for the nonattracting plates. The overall collapse is a factor of between 2 and 3 times faster for the interacting paraffin plates than for the noninteracting plates.

Another important finding, both from macroscopic theory and simulation, is that the drying phenomenon is critically sensitive to the strength of the plate-water attraction or $\epsilon$. In our previous study $^{29}$ as well as the present study we find that the critical separation for drying dramatically decreases as $\epsilon$ is increased. For large enough $\epsilon$ the contact angle of water on the surface becomes acute. In this regime macroscopic theory predicts that the critical separation for drying is zero and no cavity-induced liquid-vapor phase transition should occur. This is predicted to be the case for graphite plates, no matter how large they are. When the attractive interaction between graphite and water is scaled down, dewetting is observed, as expected from macroscopic theory. We do observe drying in relatively small paraffin plates because the paraffin-water attractive interaction is sufficiently weak that the contact angle is obtuse. In addition, the time scale for drying slows down as the strength of solutewater attractions increase.

Acknowledgment. We thank Gerhard Hummer, Claudio Margulis, Jed Pitera, Bill Swope, Ajay Royyuru, and Frank Suits for helpful comments. We also thank Montgomery Pettitt for sending us an interesting preprint of his work on graphite plates which prompted us to investigate graphite in addition to paraffin. This work was supported by a grant to B.J.B. from the National Science Foundation (CHE-00-76274) and from a SUR Grant from the IBM Corporation for a IBM LINUX CLUSTER.

\section{References and Notes}

(1) Ball, P. Nature 2003, 423, 25-26.

(2) Fersht, A. R. Structure and Mechanism in Protein Science; W. H. Freeman and Co.: New York, 1999.

(3) Brooks, C. L.; Onuchic, J. N.; Wales, D. J. Science 2001, 293, $612-613$.

(4) Dobson, C. M.; Sali, A.; Karplus, M. Angew Chem., Int. Ed. Engl. 1998, 37, 868-893.

(5) Brooks, C. L.; Gruebele, M.; Onuchic, J. N.; Wolynes, P. G. Proc. Natl. Acad. Sci. U.S.A. 1998, 95, 11037.
(6) Hummer, G.; Garde, S.; Garcia, A. E.; Pratt, L. R. Chem. Phys. 2000, 258, 349-370.

(7) Luzar, A.; Leung, K. J. Chem. Phys. 2000, 113(14), 5836-5844.

(8) Leung, K.; Luzar, A. J. Chem. Phys. 2000, 113(14), 5845-5852.

(9) Leung, K.; Luzar, A.; Bratko, D. Phys. Rev. Lett. 2003, $90(1-4)$, 65502 .

(10) Lum, K.; Luzar, A. Phys. Rev. E 1997, 56(6), 6283-6286.

(11) Pangali, C.; Rao, M.; Berne, B. J. J. Chem. Phys. 1979, 71, 29822990.

(12) Pangali, C.; Rao, M.; Berne, B. J. J. Chem. Phys. 1979, 71, 2975.

(13) Wallqvist, A.; Gallicchio, E.; Levy, R. M. J. Phys. Chem. B 2001 105, 6745-6753.

(14) Paulaitis, M. E.; Pratt, L. R. Adv. Protein Chem. 2002, 62, 283310 .

(15) Stillinger, F. H. J. Solution Chem. 1973, 2, 141.

(16) Huang, D. M.; Chandler, D. J. Phys. Chem. B 2002, 106, 20472053.

(17) Lum, K.; Chandler, D.; Weeks, J. D. J. Phys. Chem. B 1999, 103, 4570-4577.

(18) Huang, D. M.; Chandler, D. Proc. Natl. Acad. Sci. 2000, 97, 83248327.

(19) Wolde, P. R. T.; Sun, S. X.; Chandler, D. Phys. Rev. E 2001, 65, 011201.

(20) Wolde, P. R. T.; Chandler, D. Proc. Natl. Acad. Sci. 2002, 99, 6539-6543.

(21) Pratt, L. R. Annu. Rev. Phys. Chem. 2002, 53, 409-436.

(22) Talanquer, V.; Oxtoby, D. W. J. Chem. Phys. 1995, 103, 36863695.

(23) Bratko, D.; Curtis, R. A.; Blanch, H. W.; Prausnitz, J. M. J. Chem. Phys. 2001, 115, 3873-3877.

(24) Ashbaugh, H.; Paulaitis, M. J. Am. Chem. Soc. 2001, 123, 1072110728 .

(25) Truskett, T. M.; DeBenedetti, P. G.; Torquato, S. J. Chem. Phys. 2001, 114, 2401.

(26) Jensen, M. O.; Mouritsen, O. G.; Peters, G. H. J. Chem. Phys. 2004, 120, 9729-9744.

(27) Huang, D. M.; Chandler, D. Phys. Rev. E 2000, 61, 1501-1506.

(28) Huang, D. M.; Geissler, P.; Chandler, D. J. Phys. Chem. B 2001, $105,6704-6709$

(29) Huang, X.; Margulis, C. J.; Berne, B. J. Proc. Nat. Acad. Sci. U.S.A. 2003, 100, 11953-11958.

(30) Bérard, D. R.; Attard, P.; Patey, G. N. J. Chem. Phys. 1993, 98 , 7236-7244.

(31) Patey, G. N. Ber. Bunsen-Ges. Phys. Chem. 1996, 100, 885-888.

(32) Hummer, G.; Garde, S. Phys. Rev. Lett. 1998, 80, 4193-4196.

(33) Chau, P. L. Mol. Phys. 2003, 101, 3121-3128.

(34) Zhou, R.; Huang, X.; Margulius, C. J.; Berne, B. J. Science 2004, $305,1605-1609$.

(35) Allen, F. Acta Crystallogr. 2002, B58, 380-388.

(36) Berendsen, H. J. C.; Postma, J. P. M.; van Gunsteren, W. F.; Hermans, J. In Intermolecular Forces; Pullman, B., Ed.;: Reidel: Dordrecht, Holland, 1981.

(37) Gay, J.; Berne, B. J. J. Chem. Phys. 1981, 74, 3316.

(38) Wallqvist, A.; Berne, B. J. J. Phys. Chem. 1995, 99, 2893-2899.

(39) Wallqvist, A.; Berne, B. J. J. Phys. Chem. 1995, 99, 2885-2892.

(40) Jorgensen, W.; Madura, J. D.; Swenson, C. J. J. Am. Chem. Soc. 1984, 106, 6638-6646.

(41) Martyna, G. J.; Klein, M. L.; Tuckerman, M. E. J. Chem. Phys. 1992, 97, 2635

(42) Martyna, G. J.; Tobias, D. J.; Klein, M. L. J. Chem. Phys. 1994, $101,4177$.

(43) Stern, H.; Xu, H.; Harder, E.; Rittner, F.; Pavesse, M.; Berne, B. J. SIM molecular dynamics simulation program; Columbia University: New York, 2000.

(44) Andersen, H. C. J. Comput. Phys. 1983, 521, 24-34.

(45) Zhou, R.; Harder, E.; Xu, H.; Berne, B. J. J. Chem. Phys. 2001, 115,2348

(46) Luty, B. A.; Tironi, I. G.; van Gunsteren, W. F. J. Chem. Phys 1995, 103, 3014.

(47) Risken, H. The Fokker-Planck Equation; Springer: Berlin, 1989.

(48) Pettitt, B. M. Personal communication, 2004

(49) Hummer, G.; Rasaiah, J. R.; Noworyta, J. P. Nature 2001, 414, $188-190$.

(50) Lundgren, M.; Allen, N. L.; Cosgrove, T. Langmuir 2002, 18, 10462-10466. 